\title{
The employment and mental health impact of integrated Improving Access to Psychological Therapies services: Evidence on secondary health care utilization from a pragmatic trial in three English counties
}

\begin{abstract}
Objectives: Patients with a combination of long-term physical health problems can face barriers in obtaining appropriate treatment for co-existing mental health problems. This paper evaluates the impact of integrating the improving access to psychological therapies services (IAPT) model with services addressing physical health problems. We ask whether such services can reduce secondary health care utilization costs and improve the employment prospects of those so affected.
\end{abstract}

Methods: We used a stepped-wedge design of two cohorts of a total of 1,096 patients with depression and/or anxiety and comorbid long-term physical health conditions from three counties within the Thames Valley from March to August 2017. Panels were balanced. Difference-indifference models were employed in an intention-to-treat analysis.

Results: The new Integrated-IAPT was associated with a decrease of 6.15 (95\% CI: -6.84 to -5.45 ) points in the Patient Health Questionnaire-9 score and $£ 360$ (95\% CI: $-£ 559$ to $-£ 162$ ) in terms of secondary health care utilization costs per person in the first three months of treatment. The Integrated-IAPT was also associated with an $8.44 \%$ (95\% CI: $1.93 \%$ to $14.9 \%$ ) increased probability that those who were unemployed transitioned to employment.

Conclusions: Mental health treatment in care model with Integrated-IAPT seems to have significantly reduced secondary health care utilization costs among persons with long-term physical health conditions and increased their probability of employment. 


\section{Keywords}

mental health, health care utilization cost, IAPT

\section{Introduction}

One of the most under-served groups in society are those who have co-existing mental and physical health conditions. ${ }^{1-3}$ They often face barriers to accessing appropriate mental health care, ${ }^{1,2}$ which can compromise both access to - and the effectiveness of - treatments for long-term physical health conditions (LTCs), such as diabetes and cardiovascular diseases (CVDs). Additionally, they are more likely to be long-term unemployed, ${ }^{4,5}$ generating a substantial cost to the social welfare system. ${ }^{2,5}$ Co-existing mental and physical health conditions are increasingly common, and it has been estimated that in England their numbers would increase from 1.9 million in 2008 to 2.9 million in $2018 .^{5}$

Those with LTC are two to three times more likely to experience mental health problems than the general population. ${ }^{3,5}$ The cost of providing care to this group places a heavy burden on the entire health care system. Between 12 and 18\% of all British National Health Service (NHS) expenditure on LTCs (between $£ 8$ and $£ 12$ billion each year) is associated with mental illness. ${ }^{5}$ The cost of treating people with both LTCs and depression and/or anxiety disorders is about $50 \%$ higher than those who have mental health problems only. ${ }^{6}$ Moreover, these patients are less likely to be in employment than those with physical-illness alone, and the productivity of those in employment is reduced $^{7}$ or they are more likely to report sickness-absence. ${ }^{8}$ Co-morbidities also reduce economic output through their impact on premature mortality and the increasing likelihood that other family members have to take time off work to provide informal care and support.

Of the many different LTCs, diabetes is especially important. Recent estimates suggest that the number of people with diabetes worldwide will increase from 415 million in 2015 to 642 million by 2040 , with the corresponding cost of treatment expected to almost double by $2030 .^{9}$ 
Improving access to evidence-based psychological therapies might reduce health care costs, as well as accelerating the transition to employment. Both meta-analyses ${ }^{10}$ and randomized controlled trials ${ }^{11}$ report that those therapies are cost-effective. ${ }^{12}$ More recent research has investigated the effectiveness of collaborative-care, which involves a case manager working with primary-care professionals, often supervised by a mental health specialist and supported by appropriate care management. ${ }^{13}$ Much evidence, mostly from the US, shows that such care is effective. ${ }^{14,15}$ However, it is unclear whether these findings apply to those with co-existing mental and physical health conditions and whether they can be generalized to non-US settings. ${ }^{16,17}$

IAPT is the flagship programme of the NHS in England for persons with common anxiety and/or depression. Full details of the way IAPT services operate can be found in Clark. ${ }^{18}$ Briefly, IAPT operates a stepped-care delivery system. Individuals with mild to moderate depression or anxiety are often initially offered a low-intensity intervention (guided self-help, computerized cognitive behavioral therapy, psycho-education groups) delivered by a psychological wellbeing practitioner. Individuals with more severe symptoms, or those who have failed to respond to the initial low-intensity intervention, are offered high intensity treatment from an experienced mental health professional - usually hour-long, in-person, sessions once a week. Psychological wellbeing practitioners and high-intensity therapists who work in IAPT LTC services have also received training in adapting National Institute for Clinical Excellence-recommended psychological treatments for long-term conditions and in working closely with physicians. In 2018/2019 over 1 million people received IAPT. Others $(582,556)$ went on to have a multi-session course of treatment within IAPT and, among them, data on anxiety and/or depression at pre-treatment and posttreatment were available for almost all (99\%). Around half of all treated individuals (52\%) recovered and two-thirds $(67 \%)$ showed substantial reductions in their symptoms. These results are broadly in line with the outcomes one might expect from the randomized controlled trials of the relevant psychological therapies. ${ }^{20}$ The current cost for a IAPT course is $£ 684$ per patient. 
Although the IAPT programme is generally considered to be a successful example of mass implementation of evidence-based mental health care, one limitation is that people with co-morbid LTCs are under-represented there. Some reasons for this are that mental and physical illnesses tend to be managed separately and delivered in different locations. Additionally, patients with LTCs are not specifically targeted by core IAPT. In an attempt to overcome this problem, NHS England has recently encouraged the development of new Integrated-IAPT services, which focus on those who have LTCs and depression or anxiety, seeking to co-locate their physical and mental health care. ${ }^{20}$

Twenty-two early implementer sites were funded in 2016/17, with a further 15 sites funded in 2017/18. However, apart from two descriptive studies suggesting IAPT is cost-effective for those with mental health problems, ${ }^{21,22}$ cost-effectiveness has yet to be tested for specifically among persons with co-existing mental and physical health conditions. None of the previous studies was based in the geographic region considered in the current article. Moreover, the impact on employment has yet to be studied. This all makes the current study a relevant addition to current knowledge.

We report the evaluation of the Integrated-IAPT using a stepped-wedge design (SWD) of two cohorts of 1,096 patients spanning across three English counties (Berkshire, Buckinghamshire and Oxfordshire) between March 2017 and January 2018.

\section{Methods}

\section{Study design and setting}

The study took place in three neighbouring counties within the Thames Valley: Berkshire, Buckinghamshire and Oxfordshire. In each county we implemented a conventional SWD: the Integrated-IAPT provision was administered three months earlier in some areas (cohort 1) than in others (cohort 2). We collected data on secondary health care utilization (2HCU) costs from each cohort from 1 June 2017, when cohort 1 was receiving treatment, but before cohort 2 had started, 
and continued after 1 September of that year, when cohort 2 entered the scheme. In other words, the intervention group were patients in cohort 1 , who began to receive the intervention in month 0 and followed-up for three months thereafter. The control group, instead, were people in cohort 2 and they did not receive the intervention until after the three-month follow-up period for cohort 1 . Cohort 2 did eventually receive the intervention but only after our observational period had ended (for further details, see the Online Supplement, Study design).

\section{Participant eligibility criteria}

The main criteria for entry to the new Integrated-IAPT programme were identical to those for the Core-IAPT service provision (suffering from depression/ anxiety), with the additional requirement that entrants had LTCs. Each of the three counties included those LTCs that were in their models of Integrated-IAPT developed as early implementers. Thus, each included patients with both anxiety and/or depression and any of the following LTCs: asthma, chronic obstructive pulmonary disease (COPD), other respiratory conditions, CVDs, cancer, chronic fatigue syndrome, chronic musculoskeletal condition (MSDs), co-morbid diabetes (both type 1 and type 2), digestive tract condition, epilepsy, skin conditions and "others". Berkshire and Buckinghamshire also included patients with medically unexplained symptoms.

Patients had to be aged $\geq 18$ years and had an LTC. They also had to meet the established criteria for depression, on the Patient Health Questionnaire-9 (PHQ-9) or anxiety on the Generalized Anxiety Disorder-7 (GAD-7) Assessment, with, respectively, scores of $\geq 10$ or $\geq 8$; or referred for treatment for these conditions by a physician or a nurse. Patients were excluded if they were: (i) already receiving specialist mental health services; (ii) experiencing a major psychiatric condition (e.g. bipolar disorder, psychosis); (iii) posed a risk to themselves and/or others or had a cognitive impairment requiring specialist services (e.g. dementia); iv) were hospitalized for mental 
health problems; or v) were not registered with a general practitioner contracted by the Clinical Commissioning Group (the purchaser of primary health services) serving the area.

We selected all participants who: (i) survived during the study period; and (ii) had at least one Integrated-IAPT appointment during 1 June-31 August, 2017 (cohort 1) or 1 September-31 December, 2017 (cohort 2) regardless of (i) treatment; and (ii) subsequent withdrawal from treatment or deviation from the protocol. ${ }^{23}$ Therefore, we evaluated data on $2 \mathrm{HCU}$ using an intention-to-treat analysis. ${ }^{23}$ Our final sample consists of 1,096 individuals. Figure 1 in the Online Supplement presents the selection process graphically. Regarding therapy, we had complete records on delivery methods for 934 patients (582 from cohort 1 and 352 from cohort 2) during the pretreatment phase: therapy was delivered either face-to-face (116 individuals); or via telephone (816); or via telemedicine (2). However, we lacked much of the corresponding data during the treatment phase, as the delivery method was reported for only 447 patients: face-to-face (234 individuals); via telephone (182); telemedicine (3); and e-mail (28). As to patients' conditions, we have information on the initial status of 906 patients and we know that 821 were treated with low intensity and 85 with high-intensity IAPT treatment.

\section{Ethics statement}

Patients were asked to provide verbal consent for use of anonymized data at their first telephone interview with their local service. Their response was then recorded in their notes and those patients who consented to having their data tracked were included in the study. All participating IAPT services were required to submit a privacy impact assessment to their respective governance leads within the provider organizations for approval. The privacy impact assessment offers a rigorous assessment of how patient data is extracted and utilized to ensure compliance with relevant legislation. 
2HCU data were obtained from the Secondary Uses Service data at NHS health care provider level ${ }^{24}$ and were computed according to the NHS's Payment by Results scheme. These costs included attendance at emergency departments (A\&E), outpatient clinics (OP) and inpatient admissions (IP).

Depression was assessed using the nine-item Patient Health Questionnaire-9 (PHQ-9) scale, with a range from 0 to 27, where 0 represents no-depression at all and 27 indicates severe depression. Five, 10, 15 and 20 are, respectively, markers for mild, moderate, moderately severe and severe depression. ${ }^{25}$ Anxiety was assessed with the seven-item Patient Health Questionnaire Generalized Anxiety Disorder-7 (GAD-7) assessment, ranging from 0 to 21, where 0 represents noanxiety at all, and 21 represents severe anxiety. Five, 10 and 15 are, respectively, markers for mild, moderate, and severe anxiety. ${ }^{26}$

Employment status was based on self-reporting, measured in each session of IAPT, including the pre-assessment one. The counties used two different data collection systems: one system in Berkshire and a second in Buckinghamshire and Oxfordshire. Consequently, we harmonized employment codes across the two systems. Employment was categorized as: employed in full or part-time employment; unemployed and seeking work; students; long-term sick or disabled, on incapacity benefit and/or income support; unemployed but neither actively seeking for work nor receiving disability benefits; unpaid voluntary worker; retired; and declined to respond (12.2\% of the original sample). For the analysis of pathways to employment we removed individuals aged $>65$ years $(21.4 \%$ of the original sample) and individuals who declared themselves to be retired (24.4\% of the original sample or $7.15 \%$ of those younger than 66$)$, as well as students ( $2.82 \%$ of the original sample). 
Difference-in-difference and intention-to-treat analysis for secondary health care utilization

To estimate the cost of secondary care and mental health, through an intention to treat, we used linear fixed-effect models, with time point and intervention status as fixed-effects, clustered at patient level. Time-point was included as a categorical variable and intervention status as a dummy variable. Covariates, selected following discussion with clinicians, were age at start of the treatment, gender, initial PHQ-9 score, ethnicity, and treatment month. We included the former three variables as they affect clinical outcomes, whereas the last can affect both the treatment status, and the $2 \mathrm{HCU}$ because of seasonality. The effect of treatment was estimated as the interaction between the time point and the intervention status.

Since employment was recorded only at IAPT sessions, we modelled transitions in which the outcome is a dummy variable set at 1 if the individual is employed (part-time/full-time) and 0 otherwise (unemployed/long-term sick/ homemaker/ unpaid voluntary worker). Unfortunately, for 465 patients we did not know their employment status before and/or after the start of the treatment. We also excluded an additional 35 patients who were 65 or above, as employment status was not defined beyond this age. This yielded our final analytical sample of 596 individuals. The explanatory variable was a dummy corresponding to whether the individual was attending a treatment or pre-assessment session. To control for seasonality we included, as controls, the month in which each IAPT session was delivered, as well as the subject's cohort. Other covariates included age at start of treatment, gender, ethnicity, initial PHQ-9 score, current PHQ-9 and Work and Social Adjustment Scale (WSAS) score ${ }^{27}$ - a measure of impairment of functioning - and county of residence. We clustered each observation at the individual level to account for nonindependence in the sampling.

\section{Results}


Table 1 summarizes the individual characteristics during the initial baseline period (1 March-31 May, 2017) separately for treated (cohort 1) and controls (cohort 2) and by region. We compared the two cohorts in gender terms (percentage of females), age at the start of treatment, ethnicity (percentage white), and initial clinical condition: initial GAD-7, WSAS and PHQ-9 score. A t-test comparison, using unequal variance, showed differences between the treated and control groups in terms of ethnicity in Berkshire, age in Buckinghamshire, and gender in Oxfordshire.

[Table 1 about here]

Figure 1 presents the time trend in 2HCU separately for treated (blue solid line) and controls (red dashed line), adjusted for observable pre-treatment characteristics: age at start of intervention, gender, county of residency, ethnicity, and initial PHQ-9 score. Prior to enrolment, treatment and control groups exhibited similar trends, although starting from differing levels of utilization. However, following the start of treatment trends diverged. Those in the treatment group incurred fewer costs. Month 0 indicates the first 30 days from when treatment started. Consequently, a difference in the slope between treated and controls at time 0 can be attributed to enrolment.

[Figure 1 about here]

Impact of IAPT on health care utilization and costs

First, Table 2 presents the estimated results from the SWD using difference-in-difference models. In the top panel we include all the individuals in the sample. However, to ensure that our results are not distorted by a few outliers, in the bottom panel we exclude high spenders (that is, the most expensive $5 \%$ of patients: those patients who had more than $£ 10,000$ spent on them in the first quarter). Online Supplement Table S1 presents the summary statistics for this restricted group. 
Looking at the full sample (hereafter, restricted sample results are given in brackets), the control group (cohort 2) patients compared to those patients enrolled in the Integrated-IAPT (cohort 1) in June to August 2017, have a reduction of about $£ 360$ (95\% CI: $-£ 559$ to $-£ 162, p<0.001)$ ( $£ 217$ [95\% CI: $-£ 347$ to $-£ 88.7, \mathrm{p}=0.001]$ ) in total $2 \mathrm{HCU}$ per patient over the three-month period.

The largest reduction was for IP, amounting to $£ 328$ (95\% CI: $-£ 519$ to $-£ 137, \mathrm{p}=0.001$ ) ( $£ 191$ [95\% CI: $-£ 313$ to $-£ 69.3, \mathrm{p}=0.002]$ ). The reduction in A\&E was also significant, but much smaller, amounting to $£ 21.50$ (95\% CI: $-£ 31.1$ to $-£ 11.9$, p<0.001) ( $£ 16.3$ [95\% CI: $-£ 25.1$ to $£ 7.47, \mathrm{p}<0.001])$. No impact on OP was found.

[Tables 2 about here]

Next, Table 3 presents the estimated changes in depression (PHQ-9) and anxiety (GAD-7) scores during a course of treatment (defined as two or more sessions before discharge) in the IntegratedIAPT (the bottom panel presents results excluding high spenders). Looking at the full sample, we found that individuals enrolled in Integrated-IAPT experienced a reduction in their PHQ-9 score of 6.15 points (95\% CI: -6.84 to $-5.45, \mathrm{p}<0.001)(6.11$ [95\% CI: -6.81 to $-5.40, \mathrm{p}<0.001])$ and in their GAD-7 score of 4.83 points (95\% CI: -5.47 to -4.19 , p<0.001) (4.72 [95\% CI: -5.37 to -4.08 , $\mathrm{p}<0.001])$

[Table 3about here] 
Table 4 presents the estimated association between Integrated-IAPT enrolment and transition into employment for the unemployed or those outside the labour market but not retired at the initial Integrated-IAPT session in both cohorts. Integrated-IAPT enrolment was associated with a $37.5 \%$ (95\% CI: $8.68 \%$ to $66.3 \%$ ) increased probability of transitioning into employment. When we calculate the marginal effect, which represents the net average percentage of treated unemployed people who ended up employed, we found that on average $8.44 \%$ (95\% CI: 1.93 to $14.9 \%$ ) of them entered employment after starting IAPT.

[Tables 4 about here]

Robustness checks

To provide further evidence of the impact of IAPT on $2 \mathrm{HCU}$ cost, Table 5 presents the estimation results by LTC type. Here we focus on 11 specific conditions, namely: diabetes (240 patients); COPD (90); asthma (94); other respiratory conditions (3); CVDs (187); cancer (22); MSDs (62); chronic fatigue syndrome (61); epilepsy (12), chronic skin condition (9); digestive tract conditions (21); and "others" (195). For those who have multiple co-morbidities we selected the first LTC they declare. One hundred individuals (77 from cohort 1 and 23 from cohort 2) left the LTC field blank. We used a model similar to the one described above (the section headed "Difference-in-difference and intention-to-treat analysis for health care utilization") where we included a series of dummies for each of the LTCs and their interaction with the intervention status. The interaction term represents the variation in the $2 \mathrm{HCU}$ associated with being in the Integrated-IAPT group and conditional on having that specific LTC.

[Tables 5 about here] 
Our results show that the largest drop was in patients suffering from CVDs. More precisely, we found that total $2 \mathrm{HCU}$, during a course of Integrated-IAPT, dropped by $£ 882$ (95\% CI: - $£ 1884$ to $£ 120, \mathrm{p}=0.085)$. There were no significant results with other LTCs. Looking into the specific components: our results show that the largest reduction was for IP for those patients suffering from CVDs, namely $£ 955$ (95\% CI: $-£ 1888$ to $-£ 21.9, \mathrm{p}=0.045)$. The reduction in IP experienced by those patients suffering from other LTCs is less clear (10\% level only) amounted to $£ 506$ (95\% CI: -£1022 to $£ 10.9, p=0.055)$. Table 5 also shows significant reductions in terms of A\&E for patients suffering from CVDs, namely $£ 95.4$ (95\% CI: -£161 to - £30, p=0.004), COPD - 559.8 (95\% CI: $£ 119$ to $-£ 0.8, \mathrm{p}=0.045$ ), as well as digestive tract conditions, significant at the $10 \%$ level only, namely $£ 86.6$ (95\% CI: $-£ 178$ to $-£ 4.9, \mathrm{p}=0.064)$.

Online Tables 2, 3 and 4 present the estimated changes in 2HCU, depression and health care utilization for patients in caseness (i.e. patients whose PHQ-9 or GAD-7 scores, respectively, $\geq 10$ or $\geq 8$, at pre-assessment) during a course of Integrated-IAPT treatment, finding results in line with the main ones in terms of sign but of larger magnitude.

\section{Discussion}

Launched in 2008, the IAPT programme is transforming the treatment of adult anxiety disorders and depression in England. The beneficial effects on mental health care have been documented in successive annual reports from NHS Digital. ${ }^{28,29}$ However, little is known about IAPT's effects on patients' subsequent health care use. This paper fills this gap, using a SWD with 1,096 patients seen in new Integrated-IAPT services in three English counties. In line with previous research, we have shown that patients treated within Integrated-IAPT services reported an improvement in mental health, ${ }^{13}$ with a significant decrease in their PHQ-9 and GAD-7 scores. However, we help to fill a research gap ${ }^{13}$ by providing evidence of an association between Integrated-IAPT and reduced health care costs. We found that patients treated in Integrated-IAPT use less $2 \mathrm{HCU}$, with the largest share 
attributable to reduced IP and A\&E. There was no association with reduced outpatient care. In addition, we have shown an association between receipt of Integrated-IAPT and subsequent employment, with unemployed Integrated-IAPT patients more likely to find a job.

\section{Limitations}

Our study has several limitations. First, as with any study using a SWD, it is possible that the underlying trend may confound the intervention. ${ }^{30}$ This is particularly relevant given that we only have two cohorts. To address this issue, we accounted for time effects, both pre-and postintervention.

Second, our research design only allows us to assess any effects in the three months since the treatment started. Therefore, we have no evidence on the long-term effects of Integrated-IAPT.

Third, we could not examine any mechanisms that could be involved.

Fourth, our estimation is conservative, because of effect dilution due to noncompliance. We are using an intention-to-treat approach and, as such, subjects who actually did not receive any treatment, but were supposed to, were nonetheless considered part of the intervention group.

Fifth, we can only measure $2 \mathrm{HCU}$, as unfortunately we are only able to merge pre- and posttreatment primary health care use for 141 patients (12.9\% of our final sample). These individuals are self-selected and are, therefore, a potentially biased sub-group.

Sixth, we could not assess whether Integrated-IAPT is cost-effective. We avoided a costeffectiveness analysis because (i) the data is incomplete with primary and secondary health care utilization costs for only $12.8 \%$ of the sample, and no data on drug costs; and (ii) we only evaluate the short-term effects of Integrated-IAPT, and a full cost-effectiveness analysis would require a longer time horizon.

\section{Conclusions}


These findings have important implications. They show that a programme specifically tailored to individuals with LTCs can be effective. It is worth noting that our results, with their focus on improvements in depression and anxiety scores, are robust to the exclusion of the $5 \%$ most expensive patients. This may suggest that these cost differences are not related to mental health but to physical health. In parallel, we found that the results depend on different conditions: CVDs and COPD are most responsive to Integrated-IAPT intervention. Policy makers should consider this approach to reducing health care utilization among individuals with depression and/or anxiety and LTCs. Of course, further research is needed to determine how generalizable these findings are, whether the effects are sustained in the long term, whether this model of care works better with some types of patients than others, and what mechanisms are involved. We hope our findings will encourage such research. 
References - [Note to Authors: highlighted references need to be reviewed and revised; and Journal names need to be abbreviated, see https://www.ncbi.nlm.nih.gov/nlmcatalog/journals]

1. Cranwell K, Polacsek M and McCann TV. Mental health consumers' with medical comorbidity experience of the transition through tertiary medical services to primary care. International Journal of Mental Health Nursing 2016;25:127-35.

2. Firth J, Siddiqi N, Koyanagi A, et al. The Lancet Psychiatry Commission: a blueprint for protecting physical health in people with mental illness. The Lancet Psychiatry 2019;6:675-712.

3. The Lancet. Making more of multimorbidity: an emerging priority. The Lancet (London, England) 2018;391:1637.

4. Marmot M and Wilkinson R. Social determinants of health. Oxford: OUP, 2005.

5. Naylor C, Parsonage M, McDaid D, et al. Long-term conditions and mental health: the cost of co-morbidities. London, UK: The King's Fund, 2012.

6. Layard R and Clark DM. Thrive: How better mental health care transforms lives and saves money. Princeton, US: Princeton University Press, 2015.

7. Hutter N, Schnurr A and Baumeister H. Healthcare costs in patients with diabetes mellitus and comorbid mental disorders - a systematic review. Diabetologia 2010;53:2470-9.

8. Druss BG, Rosenheck RA and Sledge WH. Health and disability costs of depressive illness in a major U.S. corporation. The American journal of psychiatry 2000;157:1274-8.

9. Bommer C, Sagalova V, Heesemann E, et al. Global economic burden of diabetes in adults: projections from 2015 to 2030. Diabetes care 2018;41:963-70.

10. Chiles JA, Lambert MJ and Hatch AL. The impact of psychological interventions on medical cost offset: A meta-analytic review. Clinical Psychology: Science and Practice 1999;6:204-20.

11. Simpson JSA, Carlson LE and Trew ME. Effect of group therapy for breast cancer on healthcare utilization. Cancer practice 2001;9:19-26.

12. Blumenthal JA, Jiang W, Babyak MA, et al. Stress management and exercise training in cardiac patients with myocardial ischemia: effects on prognosis and evaluation of mechanisms. Archives of Internal Medicine 1997;157:2213-23.

13. Camacho EM, Ntais D, Coventry P, et al. Long-term cost-effectiveness of collaborative care (vs usual care) for people with depression and comorbid diabetes or cardiovascular disease: a Markov model informed by the COINCIDE randomised controlled trial. BMJ open 2016;6:e012514.

14. Katon WJ, Lin EH, Von Korff M, et al. Collaborative care for patients with depression and chronic illnesses. NEJM 2010;363:2611-20.

15. Lin EH, Katon W, Von Korff M, et al. Effect of improving depression care on pain and functional outcomes among older adults with arthritis: a randomized controlled trial. JAMA 2003;290:2428-9.

16. Jeeva F, Dickens C, Coventry P, et al. Is treatment of depression cost-effective in people with diabetes? A systematic review of the economic evidence. International journal of technology assessment in health care 2013;29:384-91.

17. Katon W, Russo J, Lin EH, et al. Cost-effectiveness of a multicondition collaborative care intervention: a randomized controlled trial. Archives of general psychiatry 2012;69:506-14.

18. Clark DM. Realizing the mass public benefit of evidence-based psychological therapies: the IAPT program. Annual Review of Clinical Psychology 2018;14:159-83.

19. NHS England. The Improving Access to Psychological Therapies Manual. 2019. https://www.england.nhs.uk/wp-content/uploads/2020/05/iapt-manual-v4.pdf (Accessed on 20/09/2020) 
20. NHS England, NHS Improvement. The Improving Access to Psychological Therapies (IAPT) pathway for people with long-term physical health conditions and medically unexplained symptoms. London, UK: NHS 2018. Available at https://wwwenglandnhsuk/publication/theimproving-access-to-psychological-therapies-iapt-pathway-for-people-with-long-term-physicalhealth-conditions-and-medically-unexplained-symptoms/ (Accessed on 11th July, 2019).

21. Mukuria C, Brazier J, Barkham M, et al. Cost-effectiveness of an improving access to psychological therapies service. The British Journal of Psychiatry 2013;202:220-7.

22. Radhakrishnan M, Hammond G, Jones PB, et al. Cost of Improving Access to Psychological Therapies (IAPT) programme: An analysis of cost of session, treatment and recovery in selected Primary Care Trusts in the East of England region. Behaviour research and therapy 2013;51:37-45. 23. Gupta SK. Intention-to-treat concept: a review. Perspectives in clinical research 2011;2:109.

24. NHS England. Secondary Uses Service (SUS). In: (RBAC) R-BAC, editor. 2017. 25. Kroenke K, Spitzer RL and Williams JB. The PHQ-9: validity of a brief depression severity measure. Journal of general internal medicine 2001;16:606-13.

26. Löwe B, Decker O, Müller S, et al. Validation and standardization of the Generalized Anxiety Disorder Screener (GAD-7) in the general population. Medical care 2008;46:266-74.

27. Mundt JC, Marks IM, Shear MK, et al. The Work and Social Adjustment Scale: a simple measure of impairment in functioning. The British Journal of Psychiatry 2002;180:461-4.

28. Digital N. Psychological Therapies: reports on the use of IAPT services, England - June 2018 final, including reports on the IAPT pilots and quarter 1 2018-19 data. 2018.

29. Digital N. Psychological Therapies: reports on the use of IAPT services, England March 2019 including reports on the IAPT pilots and Quarter 4 2018-19 data. 2019.

30. Hemming K, Haines TP, Chilton PJ, et al. The stepped wedge cluster randomised trial: rationale, design, analysis, and reporting. BMJ 2015;350:h391. 
Figure 1. Time-trend in $2 \mathrm{HCU}$ by treatment month, by cohort (adjusted)

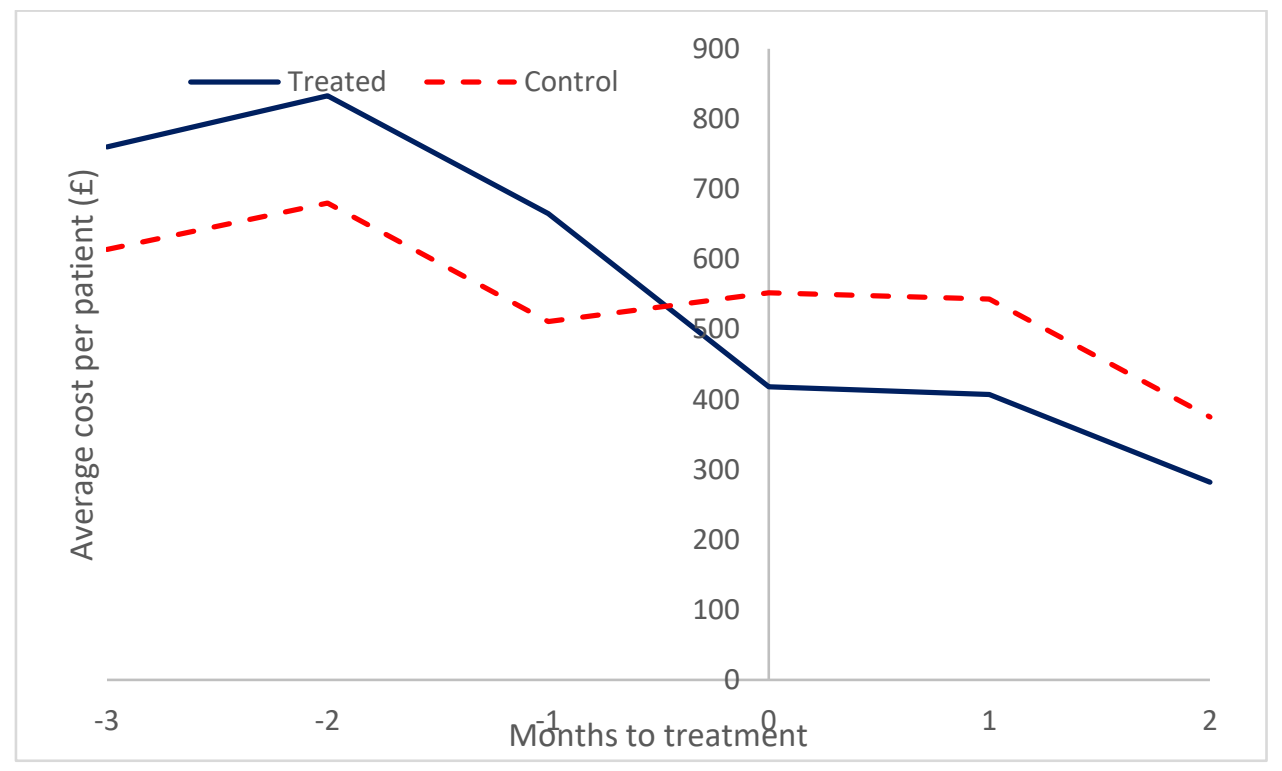

Notes: The time horizon refers to 1 March-30 August, 2017. "Treated" represents individuals enrolled in cohort 1. They started the treatment between 1 June-31 August, 2017. "Controls" refers to the individuals enrolled in cohort 2. Our analytical sample was restricted to individuals who receive at least one Integrated-IAPT session during the treatment period and did not die during the trial. The outcome represents the average secondary health care utilization per patient $(\mathfrak{f})$. The outcome was adjusted for individual characteristics, namely age at start of the trial, gender, county of residency, ethnicity, initial PHQ score. With month 0 we indicate the first month following the beginning of the treatment. 
Table 1. Baseline comparison including high spenders

\begin{tabular}{|c|c|c|c|}
\hline \multirow[b]{2}{*}{ Covariates } & Treated & \multicolumn{2}{|l|}{ Controls } \\
\hline & Mean & Mean & $\begin{array}{c}\text { T-test } \\
\text { comparison }\end{array}$ \\
\hline Berkshire & & & \\
\hline Female (percentage) & $54.8 \%$ & $53.8 \%$ & 0.20 \\
\hline Age (years) & 55.8 & 55.1 & 0.44 \\
\hline White (percentage) & $83.5 \%$ & $93.5 \%$ & $-3.40 * * *$ \\
\hline Initial GAD-7 score & 11.7 & 11.5 & 0.27 \\
\hline Initial WSAS score & 6.88 & 6.36 & 1.60 \\
\hline Initial PHQ-9 score & 14.6 & 14.3 & 0.34 \\
\hline
\end{tabular}

\section{Buckinghamshire}

Female (percentage)

$63.4 \% \quad 69.0 \% \quad-1.20$

Age (years)

$\begin{array}{lll}49.2 & 52.5 & -1.98 * *\end{array}$

White (percentage)

$\begin{array}{lll}86.1 \% & 93.8 \% & 1.48\end{array}$

Initial GAD-7 score

12.5

$-0.06$

Initial WSAS score

6.18

3.25

1.48

Initial PHQ-9 score

$14.9 \quad 10.2$

1.14

\section{Oxfordshire}

Female (percentage)

$53.1 \% \quad 62.4 \% \quad-2.18 * *$

Age (years)

55.9

52.9

1.95*

White (percentage)

$94.8 \% \quad 93.9 \% \quad-0.47$


Initial GAD-7 score

Initial WSAS score

Initial PHQ-9 score

Number of observations

$\mathrm{P}$-value is calculated using two-tailed t-test.

$* \mathrm{p}<0.10, * * \mathrm{p}<0.05, * * * \mathrm{p}<0.01$

Statistically significant differences are in bold. $\begin{array}{lll}12.4 & 12.3 & 0.10\end{array}$

$\begin{array}{lll}6.45 & 6.58 & -0.47\end{array}$

$\begin{array}{lll}14.9 & 15.3 & -0.72\end{array}$

$598 \quad 498$ 
Table 2. Estimates of treatment effect from a stepped-wedge design model on the impact of using Integrated-IAPT therapy on $2 \mathrm{HCU}$

\begin{tabular}{lcccc}
\hline & Variation in total & Variation in & Variation in & Variation in \\
& secondary health & A\&E cost & inpatient & outpatient \\
& care costs per patient & (p.p.) & cost & cost \\
& (p.p.) & & (p.p.) & (p.p.) \\
\hline Treated with IAPT & $\mathbf{- \$ 3 6 0 * * *}$ & $\mathbf{- £ 2 1 . 5 * * *}$ & $\mathbf{- \$ 3 2 8 * *}$ & $-£ 11.3$ \\
95\% confidence intervals & {$[-559,-162]$} & {$[-31.1,-11.9]$} & {$[-519,-137]$} & {$[-31.5,8.95]$} \\
Months fixed-effects & Yes & Yes & Yes & Yes \\
\hline N of patients & 1,096 & 1,096 & 1,096 & 1,096 \\
\hline
\end{tabular}

Sensitivity test: results excluding the most expensive $5 \%$ of patients

\begin{tabular}{lcccc}
\hline & Variation in total & Variation in & Variation in & Variation in \\
& secondary health & A\&E cost & inpatient cost & outpatient cost \\
& care costs (p.p.) & (p.p.) & (p.p.) & (p.p.) \\
\hline Treated with IAPT & $\mathbf{- £ 2 1 7 * *}$ & $\mathbf{- £ 1 6 . 3 * * *}$ & $\mathbf{- £ 1 9 1 * *}$ & $-£ 10.2$ \\
$95 \%$ confidence intervals & {$[-347,-88.7]$} & {$[-25.1,-7.47]$} & {$[-313,-69.3]$} & {$[-30.2,9.75]$} \\
Months fixed-effects & Yes & Yes & Yes & Yes \\
\hline N of patients & 1,060 & 1,060 & 1,060 & 1,060
\end{tabular}

Notes: ${ }^{\mathrm{p}}<0.1, * * \mathrm{p}<0.05, * * * \mathrm{p}<0.01$. Statistically significant differences are in bold. Estimation results from a within-group estimation with robust standard error. The time horizon refers to 1 March-31 August, 2017. The outcome variable represents the total secondary care cost, the A\&E, the Inpatient and Outpatient one (expressed in $£$ per patient (p.p.)). The explanatory variable represents whether the individual has been enrolled and received at least one Integrated-IAPT session. "Treated" represents individuals enrolled in cohort 1 - they started the treatment between 1 June-31 August, 2017. "Controls" refers to the individuals enrolled in cohort 2 . We controlled for time-period effects, expressed in fulltime equivalent months. In the sensitivity test we excluded patients whose total secondary care expenditure was higher than $£ 10,000$. 
Table 3. Estimates of treatment effect from a stepped-wedge design model on the impact of using Integrated-IAPT therapy on mental health

\begin{tabular}{lcc}
\hline & Variation in & Variation in \\
& PHQ-9 & GAD-7 \\
& score & score \\
\hline Treated with IAPT & $\mathbf{- 6 . 1 5 * * *}$ & $\mathbf{- 4 . 8 3 * * *}$ \\
$95 \%$ confidence intervals & {$[-6.84,-5.45]$} & {$[-5.47,-4.19]$} \\
Months fixed-effects & Yes & Yes \\
\hline N of patients & 916 & 916 \\
\hline
\end{tabular}

Sensitivity test: results excluding the most expensive $5 \%$ of patients

\begin{tabular}{lcc}
\hline & Variation in & Variation in \\
& PHQ-9 & GAD-7 \\
& score & score \\
\hline Treated with IAPT & $\mathbf{- 6 . 1 1 * * *}$ & $\mathbf{- 4 . 7 2 * * *}$ \\
$95 \%$ confidence intervals & {$[-6.81,-5.40]$} & {$[-5.37,-4.08]$} \\
Months fixed-effects & Yes & Yes \\
\hline N of patients & 885 & 885
\end{tabular}

Notes: ${ }^{\mathrm{p}}<0.1, * * \mathrm{p}<0.05, * * * \mathrm{p}<0.01$. Statistically significant differences are in bold. Estimation results from a within-group estimation with robust standard error. The time horizon refers to 1 March-31 August, 2017. The outcome variable represents the PHQ-9 and GAD-7 score. Higher score represents worse mental health. The explanatory variable represents whether the individual had been enrolled and received at least two Integrated-IAPT session. "Treated" represents individuals enrolled in cohort 1 . They started the treatment between 1 June-31 August, 2017. "Controls" refers to the individuals who were observed in the pre-assessment session. We controlled for time periods, expressed in fulltime equivalent months. In the sensitivity test we excluded patients whose total secondary care expenditure was higher than $£ 10,000$. 
Table 4. Estimates of treatment effect using a linear probability model, on the impact of using Integrated-IAPT therapy on transit into employment, including high spenders

\begin{tabular}{cc}
\hline Transition from & Transition from \\
unemployment into & unemployment into \\
employment & employment \\
probability & probability, \\
& marginal effects \\
\hline
\end{tabular}

Treated with IAPT

$0.37 * *$

$0.08 * *$

$95 \%$ confidence intervals

$[0.02,0.15]$

$[0.9,0.66]$

Months fixed-effects

Yes

Yes

$\mathrm{N}$ of patients

596

596

Notes: ${ }^{\mathrm{p}}<0.1,{ }^{* *} \mathrm{p}<0.05,{ }^{* * *} \mathrm{p}<0.01$. Statistically significant differences are in bold. Estimation results from a logit estimation with robust standard error clustered at individual level. The time horizon refers to 1 March-31 August, 2017. The outcome is the marginal effect, which represents the net average percentage of treated unemployed people who ended up employed (in other words, the number of people moving into employment minus the number moving out of employment). "Treated" is a dummy variable equal to 1 whether the individual has started the Integrated-IAPT treatment and 0 if the individual is observed in pre-assessment. We controlled for time-period effects. 
Table 5. Estimates of treatment effect from a stepped-wedge design model on the impact of using Integrated-IAPT therapy on 2HCU: sensitivity test by type of LTC

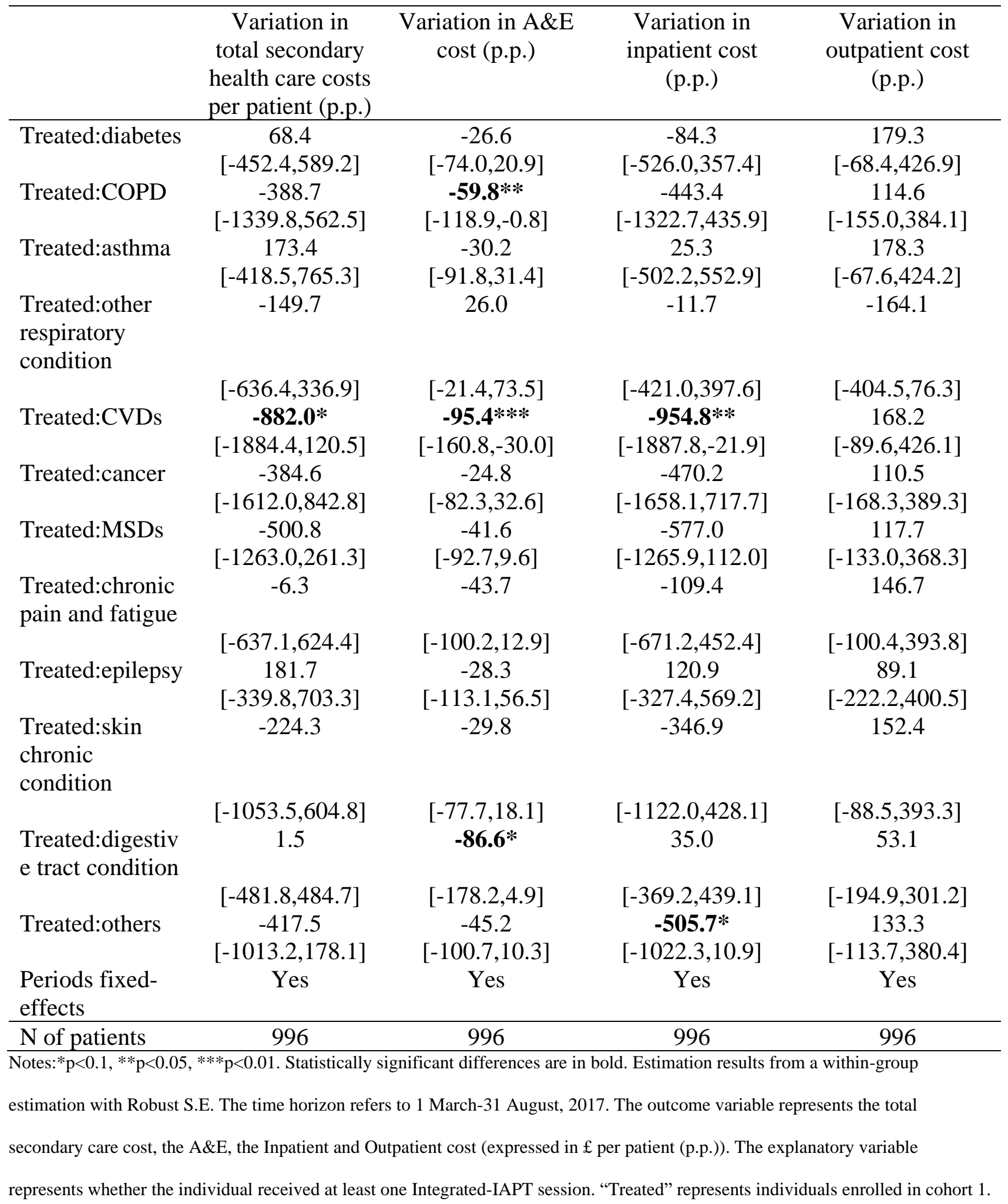


They started treatment between 1 June-31 August, 2017. "Controls" refers to the individuals enrolled in cohort 2. We controlled for time-periods effects, expressed in fulltime equivalent months. 